\title{
Efectos del transporte marítimo en contenedores sobre el crecimiento económico en los países de la costa oeste de América Latina
}

\author{
María Jesús Freire-Seoane, Beatriz López-Bermúdez \\ e Ignacio de la Peña Zarzuelo
}

\section{Resumen}

Se analizan los efectos producidos por cambios en el transporte marítimo en contenedores, el nivel de desempleo, el nivel de competitividad y los tratados comerciales sobre el PIB per cápita de los países de la costa oeste de América Latina. La muestra utilizada se compone de 23 puertos de esta costa y 8 observaciones temporales (2008 a 2015). La estimación con datos de panel se realiza para efectos fijos básicos, efectos fijos (robust) y el modelo de errores estándar corregidos para panel (PCSE) con las características propias de la muestra. En esta investigación, la estimación que ofrece mejores resultados es el modelo PCSE. Los resultados respaldan la utilización del volumen de mercancía en contenedores como una variable significativa del crecimiento económico; además, se pone de manifiesto la necesidad de realizar inversiones en infraestructuras portuarias y continuar con la puesta en marcha de instrumentos de política comercial de carácter aperturista.

\section{Palabras clave}

Transporte marítimo, transporte por contenedores, competitividad, desempleo, acuerdos económicos, modelos econométricos, política de transporte, crecimiento económico, América Latina

\section{Clasificación JEL}

C33, F63, N76

\section{Autores}

María Jesús Freire-Seoane es Profesora Emérita en la Facultad de Economía y Empresa de la Universidad de La Coruña (España). Correo electrónico: maje@udc.es.

Beatriz López-Bermúdez es Profesora en la Facultad de Economía y Empresa de la Universidad de La Coruña (España). Correo electrónico: beatriz.lopez2@udc.es.

Ignacio de la Peña Zarzuelo es Profesor Asociado en el Departamento de Ingeniería Civil de la Universidad Politécnica de Madrid (España). Correo electrónico: i.delapena@upm.es. 


\section{Introducción}

La globalización ha supuesto la unión de los países y el incremento del comercio de tal modo que las únicas barreras existentes en la actualidad son resultado de las diferentes políticas económicas, que en algunos casos pueden ser fuertemente restrictivas. Además, la globalización ha dado lugar a que las economías que adoptan una logística eficiente puedan crecer más rápido, ser más competitivas e incrementar su inversión. Esta hipótesis lleva a considerar que el desempeño logístico es uno de los factores claves del comercio internacional.

Resulta evidente que la logística del transporte marítimo es un asunto de carácter puramente transversal, que, por una parte, comprende la producción, el comercio, el desarrollo empresarial, el sector del transporte, las tecnologías de la información y las comunicaciones, el control de las mercancías y las facilidades del transporte y el comercio y, por otra, también incluye las diversas entidades involucradas en todo el proceso. En este sentido, a los Gobiernos no les faltan motivos para comprometerse en el desarrollo de un sistema logístico más eficiente, tanto para promover la competitividad de las exportaciones y de las infraestructuras nacionales, como para incentivar el comercio internacional, ganar nuevos mercados e impulsar el empleo tanto en el sector de los servicios como en las empresas auxiliares. Los Gobiernos también tratan de atenuar las externalidades ambientales y sociales que generan la congestión, la accidentabilidad, la inseguridad y la contaminación de la actividad portuaria (Cipoletta Tomassian, Pérez y Sánchez, 2010).

Históricamente los países latinoamericanos han mostrado en sus relaciones comerciales una gran dependencia de las economías desarrolladas, sobre todo de los Estados Unidos, la Unión Europa y, más recientemente, la región de Asia y el Pacífico. En los últimos años, los países asiáticos se han posicionado como grandes socios comerciales de América Latina. Para seguir manteniendo estos vínculos mercantiles, las economías de la costa oeste de América Latina han tenido que desarrollar instrumentos comerciales que faciliten las relaciones de intercambio, que, por otra parte, son fundamentales para el desarrollo de la región (Bernal-Meza, 2015; Girón, 2015).

El comercio ha sido uno de los motores del crecimiento experimentado en América Latina y el Caribe en los últimos años. En particular, en los países de la costa oeste de América Latina el aumento de la demanda internacional de materias primas, sobre todo de la región de Asia y el Pacífico, provocó entre 2009 y 2011 importantes incrementos de sus precios, que llevaron aparejado un crecimiento generalizado del producto interno bruto (PIB) y de la productividad laboral, así como una incipiente y sostenida política de integración regional (CEPAL, 2014).

El objetivo principal de este estudio es determinar el efecto producido por cambios en el transporte marítimo en contenedores, el nivel de desempleo, el nivel de competitividad y los tratados comerciales sobre el PIB per cápita de los países de la costa oeste de América Latina en el período comprendido entre 2008 y 2015.

El artículo se divide en cinco secciones, la primera de las cuales es esta introducción. En la segunda, se revisan los antecedentes proporcionados por los estudios sobre crecimiento económico y transporte marítimo; en la tercera, se presenta la metodología de la investigación (en el primer apartado se indican las variables que se utilizarán en el análisis y en el segundo se describe el modelo econométrico que se aplicará en el análisis empírico); en la cuarta sección se exponen los resultados obtenidos de la estimación y, por último, en la quinta sección se presentan las conclusiones. 


\section{Antecedentes}

La teoría general sobre las relaciones entre crecimiento económico y comercio es la base de la teoría del crecimiento (Rivera-Batiz y Romer, 1991; Romer, 1990), pero también existen algunas reformulaciones a nivel teórico originadas por la globalización (Bolaky y Freund, 2004; Grossman y Helpman, 1993). Este tipo de aproximaciones comenzaron a desarrollarse inicialmente en los trabajos de Radelet y Sachs (1998) y Redding (2002), y se convirtieron a partir de 2007 en un elemento clave del informe anual sobre transporte marítimo Review of Maritime Transport que publica la Conferencia de las Naciones Unidas sobre Comercio y Desarrollo (UNCTAD, s/fa). Sin embargo, los primeros trabajos de investigación basados en especificaciones empíricas son relativamente recientes, sobre todo en lo relativo a la relación entre actividad portuaria y crecimiento económico (Grossmann y otros, 2007).

La investigación de Corbett y Winebrake (2008) demuestra que el tráfico portuario en contenedores está correlacionado con el crecimiento. En la actualidad, el estudio de las dinámicas entre el PIB y los indicadores de movimiento portuario es un elemento crucial tanto para la estrategia comercial de los actores económicos que participan en el negocio marítimo (The Maritime Executive, 2014; Rodrigue y otros, 2010) como para los investigadores en economía marítima, que centran sus esfuerzos en calcular factores adicionales que completen y refuercen la vinculación entre el PIB y la actividad portuaria. Otros estudios más recientes que miden el impacto del tráfico marítimo en el crecimiento y el desarrollo de los países son los de Bernhofen, El-Sahli y Kneller (2016) y Rodrigue, Comtois y Slack (2013). Algunos autores se centran en la relación entre costo y distancia del transporte (Radelet y Sachs, 1998) y otros en los costos del transporte y los acuerdos transfronterizos de cooperación (Micco y Pérez, 2001); por último, también se investiga el grado de especialización industrial (Redding, 2002).

En 2004, la Comisión Económica para América Latina y el Caribe (CEPAL) realizó un primer intento de resaltar la importancia económica de las infraestructuras portuarias de América Latina y el Caribe en el complejo y cambiante sistema global de rutas marítimas. El objetivo del estudio incluía el análisis de la oferta y la demanda de servicios marítimos, el precio de los fletes, la propiedad de la flota y el régimen de gobernanza portuaria (Sánchez, 2004). En las investigaciones de la UNCTAD (s/fb) y de Fay y Morrison (2007) también se pone de relieve el papel central que América Latina podría desempeñar respecto de los patrones internacionales del tráfico marítimo. En estos trabajos se plantea de modo explícito la necesidad de aprovechar el impulso económico de los precios altos de las materias primas, ventaja que para muchos autores no ha sido aprovechada en términos de reducción de la desigualdad y aumento de la calidad y la eficiencia de las infraestructuras (Bitar, 2016).

Sánchez y otros (2015) analizan los desafíos y oportunidades que el transporte marítimo y el desarrollo portuario suponen para América Latina y el Caribe, introduciendo el tema de la sostenibilidad como eje transversal de las mejoras de las infraestructuras. Afirman que, además de las mejoras en infraestructuras, es importante señalar la importancia de otros factores, como las características de la terminal portuaria o el sistema de gobernanza, que muchos autores han investigado en relación con el concepto de eficiencia (Serebrisky y otros, 2016; Chang y Tovar, 2014; Núñez-Sánchez y Coto-Millán, 2012; Ramos-Real y Tovar, 2010; Coto-Millán, Baños-Pino y Rodríguez-Álvarez, 2000; Roll y Hayuth, 1993).

En el trabajo de López-Bermúdez, Freire y Pais (2018), se muestra que los proyectos de ampliación de terminales de portacontenedores en los países pertenecientes a la Alianza del Pacífico y el Mercado Común del Sur (MERCOSUR) presentan un efecto sobre el PIB per cápita en dólares constantes de 2011 en paridad del poder adquisitivo del 16,22\% y el 11,42\%, respectivamente. 


\section{Metodología}

\section{Variables}

Las variables consideradas en el análisis son: el PIB per cápita, el volumen de mercancía movido expresado en unidades de TEU (unidad equivalente a 20 pies), la tasa de desempleo, el índice de competitividad y los tratados comerciales. En el cuadro 1 se detallan las variables empleadas en el análisis.

\section{Cuadro 1}

Variables utilizadas en el modelo

\begin{tabular}{llll}
\hline Tipo de variable & \multicolumn{2}{l}{ Variable } & Unidad de medida \\
\hline Dependiente & $Y$ & PIB per cápita & Dólares constantes de 2011 en paridad del poder adquisitivo \\
\hline Independiente & $X_{1}$ & Volumen de los contenedores & Unidad equivalente a 20 pies (TEU) \\
\cline { 2 - 4 } & $X_{2}$ & Tasa de desempleo & Porcentaje \\
\cline { 2 - 4 } & $X_{3}$ & Índice de competitividad & Índice \\
\hline$X_{4}$ & Tratados comerciales & Variable ficticia (dummy) \\
\hline
\end{tabular}

Fuente: Elaboración propia.

\section{a) PIB per cápita}

El PIB recoge la producción de bienes y servicios de un país durante un período de tiempo determinado, por norma general un año. Es la variable económica por excelencia que se utiliza como reflejo del grado de prosperidad o recesión interna del país y se emplea habitualmente como un indicador de su nivel de riqueza, sobre todo, en el análisis internacional, cuando se expresa en dólares de un año base y en paridad del poder adquisitivo.

El PIB per cápita se ha calculado como el cociente entre el PIB y la población total del país en cada uno de los años analizados. Con el fin de poder comparar diferentes economías, es necesario disponer de un valor homogeneizado, eliminando, entre otros factores, los efectos de las monedas extranjeras y la inflación. Por este motivo se utiliza el PIB per cápita en paridad del poder adquisitivo (PPP) y expresado en dólares constantes de 2011 (Banco Mundial, s/f).

En el cuadro 2 se presentan los valores del PIB per cápita expresados en PPP y en dólares constantes de 2011, en el período comprendido entre 2008 y 2015, de los diez países donde se localizan los 23 puertos objeto de análisis. Los valores oscilan entre un máximo de 22.537 dólares en 2015 en Chile y un mínimo de 3.907 dólares en 2009 en Nicaragua. El crecimiento promedio del PIB per cápita del conjunto de países durante el período analizado fue del 18,27\%, es decir, el promedio anual fue del $2,28 \%$.

\section{Cuadro 2}

América Latina (10 países): PIB per cápita, 2008-2015

(En dólares constantes de 2011 en paridad del poder adquisitivo)

\begin{tabular}{lcccccccc}
\hline & 2008 & 2009 & 2010 & 2011 & 2012 & 2013 & 2014 & 2015 \\
\hline Chile & 19032 & 18547 & 19442 & 20438 & 21330 & 21998 & 22226 & 22537 \\
\hline Colombia & 10547 & 10600 & 10901 & 11496 & 11840 & 12296 & 12716 & 12985 \\
\hline Costa Rica & 12835 & 12544 & 13000 & 13397 & 13878 & 14035 & 14392 & 14914 \\
\hline Ecuador & 9286 & 9184 & 9352 & 9927 & 10322 & 10665 & 10923 & 10777 \\
\hline
\end{tabular}


Cuadro 2 (conclusión)

\begin{tabular}{lrrrrrrrr}
\hline & \multicolumn{1}{c}{2008} & \multicolumn{1}{c}{2009} & 2010 & 2011 & 2012 & 2013 & \multicolumn{1}{c}{2014} & 2015 \\
\hline El Salvador & 7501 & 7234 & 7300 & 7428 & 7533 & 7636 & 7707 & 7845 \\
\hline Guatemala & 6782 & 6670 & 6714 & 6844 & 6899 & 7005 & 7147 & 7293 \\
\hline México & 16008 & 15012 & 15535 & 15923 & 16324 & 16316 & 16459 & 16668 \\
\hline Nicaragua & 4092 & 3907 & 4029 & 4231 & 4453 & 4619 & 4785 & 4961 \\
\hline Panamá & 14867 & 14839 & 15419 & 16940 & 18184 & 19057 & 19872 & 20674 \\
\hline Perú & 9323 & 9309 & 9957 & 10449 & 10944 & 11430 & 11545 & 11768 \\
\hline
\end{tabular}

Fuente: Elaboración propia, sobre la base de datos del Banco Mundial, "Indicators", s/f [en línea] https://data.worldbank.org/ indicator?tab=all.

\section{b) Volumen de los contenedores}

La medición del efecto del transporte marítimo en la economía de un país puede realizarse a través de diferentes indicadores. En esta investigación se utiliza la mercancía transportada en contenedores, porque es la que presenta un mayor valor añadido en el mercado de bienes y servicios, de modo que, en principio, genera importantes beneficios que mejoran el nivel de vida de la sociedad.

El desarrollo del transporte multimodal y la práctica del transporte de mercancía en contenedores son dos factores que se han desarrollado a la par en un mismo período temporal, lo que ha redundado en que el análisis de la mercancía transportada en contenedores sea un fiel reflejo del desarrollo de las infraestructuras de la cadena logística de consumo. Por una parte, la práctica del transporte en contenedores y el aprovechamiento de las economías de escala se han traducido en las grandes proporciones de los buques portacontenedores que, en la actualidad, llegan hasta 24.000 TEU. Por otra parte, el protagonista del transporte intermodal es, indudablemente, el contenedor, que permite un fácil manejo en los sistemas modales (Rodrigue, Comtois y Slack, 2013).

El volumen de mercancía movido en el período comprendido entre 2008 y 2015 en cada uno de los puertos considerados de la costa oeste de América Latina se expresa en unidades de TEU (CEPAL, $s / f)$.

LOS TEU movidos en los puertos se incluyen en el modelo econométrico como un indicador, ya que al realizar las estimaciones las variables deben estar expresadas en las mismas unidades y, por ello, no se han utilizado los valores absolutos (Guisan, 2008). La variable TEU está expresada en miles y los valores oscilan entre un máximo de 8.151 en el puerto de Balboa (Panamá) y un mínimo de 0 en el puerto de Coronel (Chile).

En el gráfico 1 se muestra la evolución del movimiento de carga en contenedores en el período comprendido entre 2008 y 2015. Se ha calculado un índice tomando 2008 como año base con un valor de 100. Sobre la base de la información disponible se observa que en 2009 el movimiento de contenedores se redujo 12 puntos porcentuales, alcanzando el valor de 88. Sin embargo, a partir de 2010 se inicia en esta área geográfica una senda de crecimiento, que presenta oscilaciones, pero siempre con variaciones de signo positivo. En 2010 el índice de TEU movidos alcanza el valor de 106,27 con una tasa de crecimiento del 20,46\%; en 2011 la tasa de crecimiento sigue siendo de dos dígitos y alcanza el 17,92\%; en 2012 el valor absoluto sigue subiendo y llega hasta 134, no obstante la tasa de crecimiento disminuye al 7,2\%; el proceso de declive de la tasa de crecimiento se mantiene en 2013, cuando dicha tasa alcanza el 2,23\%; sin embargo, en los últimos años analizados los valores absolutos se incrementan hasta 147 en 2014 y 156 en 2015, con tasas de crecimiento del 7,30\% y el $6,12 \%$, respectivamente. 


\section{Gráfico 1}

América Latina (10 países): índice de TEU, 2008-2015ª

(Año base $2008=100$ )

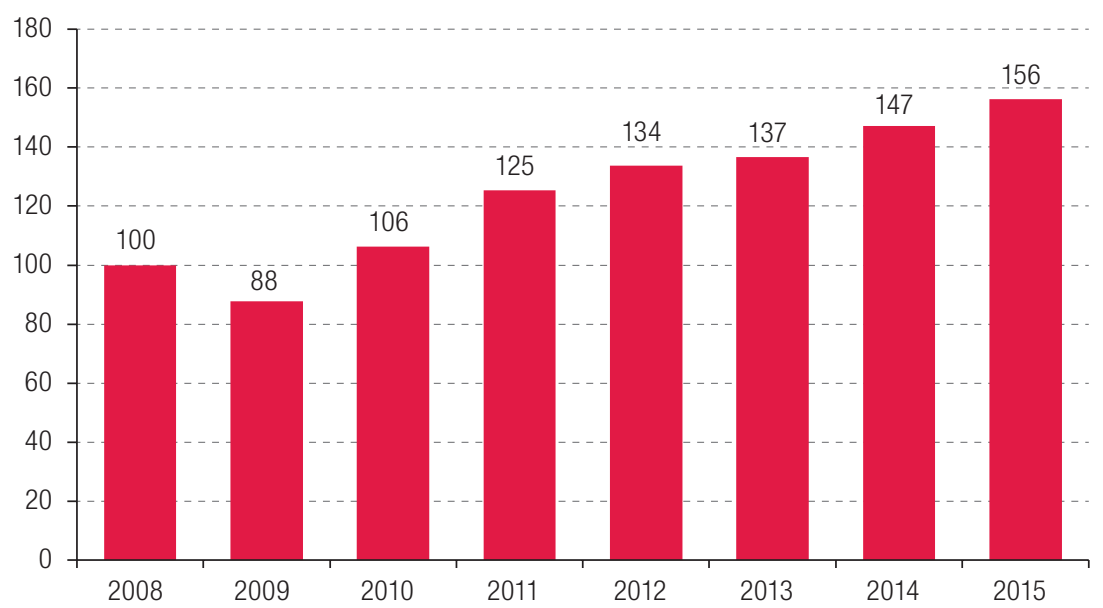

Fuente: Elaboración propia, sobre la base de datos de Comisión Económica para América Latina y el Caribe (CEPAL), "Perfil marítimo y logístico de América Latina y el Caribe", s/f [en línea] http://bit.ly/2fV5bzS.

a TEU: unidad equivalente a 20 pies. Los países incluidos son: Chile, Colombia, Costa Rica, Ecuador, El Salvador, Guatemala, México, Nicaragua, Panamá y Perú.

\section{c) Tasa de desempleo}

La tasa de desempleo se expresa como el porcentaje de población activa de cada uno de los países objeto de estudio que está buscando empleo y no lo encuentra en cada uno de los años considerados (Banco Mundial, s/f). La tasa de desempleo refleja la situación del mercado laboral.

En el cuadro 3 se muestra la tasa de desempleo de estos países en el período comprendido entre 2008 y 2015. El valor máximo es del 12,07\% en 2009 en Colombia y el mínimo del 2,79\% en 2008 en Guatemala, si no se considera el último año de la serie. En 2015 la mayor tasa de desempleo tuvo lugar en Costa Rica y fue del 9,61\%, mientras que Guatemala siguió mostrando el valor mínimo, del 2,42\%.

Cuadro 3

América Latina (10 países): tasa de desempleo, 2008-2015

(En porcentajes)

\begin{tabular}{lrrrrrrrr}
\hline & 2008 & 2009 & 2010 & 2011 & 2012 & 2013 & 2014 & 2015 \\
\hline Chile & 7,80 & 9,69 & 8,14 & 7,12 & 6,43 & 5,93 & 6,39 & 6,21 \\
\hline Colombia & 11,27 & 12,07 & 11,83 & 10,88 & 10,43 & 9,70 & 9,15 & 8,95 \\
\hline Costa Rica & 4,78 & 7,71 & 8,92 & 10,31 & 10,17 & 9,38 & 9,62 & 9,61 \\
\hline Ecuador & 7,30 & 6,47 & 5,02 & 4,21 & 4,12 & 4,15 & 3,80 & 4,77 \\
\hline El Salvador & 5,88 & 7,33 & 7,05 & 6,62 & 6,07 & 5,93 & 5,92 & 6,18 \\
\hline Guatemala & 2,79 & 3,08 & 3,74 & 4,13 & 2,87 & 2,99 & 2,91 & 2,42 \\
\hline México & 3,90 & 5,38 & 5,33 & 5,19 & 4,92 & 4,94 & 4,83 & 4,34 \\
\hline Nicaragua & 6,20 & 8,20 & 8,00 & 7,42 & 6,74 & 5,30 & 5,27 & 5,61 \\
\hline Panamá & 5,60 & 6,60 & 6,50 & 4,48 & 4,05 & 4,10 & 4,82 & 5,35 \\
\hline Perú & 6,64 & 4,40 & 4,00 & 3,90 & 3,60 & 4,00 & 4,08 & 4,42 \\
\hline
\end{tabular}

Fuente: Elaboración propia, sobre la base de datos del Banco Mundial, "Indicators", s/f [en línea] https://data.worldbank.org/ indicator?tab=all. 


\section{d) Índice de competitividad}

El índice mundial de competitividad (Global Competitiveness Index (GCI)) lo elabora y publica el Foro Económico Mundial (Schwab, 2016) y su finalidad es mostrar el nivel de competitividad de cada país sobre la base de una escala común en todo el mundo. El rango de este indicador abarca entre 1 y 7 , donde 1 es el valor más bajo y 7 el más alto. Para obtener este indicador, se analizan diferentes aspectos del país agrupados en doce pilares, que son:

- Pilar 1: Instituciones

- Pilar 2: Infraestructuras

- Pilar 3: Entorno macroeconómico

- Pilar 4: Salud y educación primaria

- Pilar 5: Educación superior y formación

- Pilar 6: Eficiencia en el mercado de bienes

- Pilar 7: Eficiencia del mercado laboral

- Pilar 8: Desarrollo del mercado financiero

- Pilar 9: Preparación tecnológica

- Pilar 10: Tamaño del mercado

- Pilar 11: Sofisticación en materia de negocios

- Pilar 12: Innovación

En términos generales, se puede afirmar que en 2016 el mayor valor del índice mundial de competitividad lo tiene Suiza, con un 5,81. Dentro del grupo de países analizados, el primer lugar corresponde a Chile, con un 4,64, que alcanza el puesto 33 a nivel internacional, seguido de Panamá, con un 4,51 y la posición 42 .

En el cuadro 4 se presenta el índice de competitividad de los países objeto de este estudio en el período comprendido entre 2008 y 2015. En 2008 los valores oscilan entre el más alto, de 4,75, correspondiente a Chile, y el más bajo, de 3,32, correspondiente a Nicaragua. A lo largo de los años analizados el indicador mejora en casi todos los países, con la excepción de Chile, en que paulatinamente empeora, hasta alcanzar un valor de 4,60 en 2015; a pesar de ello, este es el país que se encuentra en las mejores condiciones en términos relativos. En 2015 Nicaragua sigue siendo el país que muestra el valor más bajo del índice de competitividad; no obstante, su situación ha mejorado de forma continua, de modo que su índice alcanza un valor de 3,82. Entre estos datos del índice máximo (Chile) y mínimo (Nicaragua) se encuentran el resto de los países, en los que el indicador varía entre 4,01 en el caso de El Salvador y 4,43 en el caso de Panamá.

\section{Cuadro 4}

América Latina (10 países): índice de competitividad, 2008-2015 (En valores en una escala de 1 a 7 )

\begin{tabular}{lllllllll}
\hline & 2008 & 2009 & 2010 & 2011 & 2012 & 2013 & 2014 & 2015 \\
\hline Chile & 4,75 & 4,70 & 4,72 & 4,69 & 4,70 & 4,65 & 4,61 & 4,60 \\
\hline Colombia & 4,03 & 4,05 & 4,05 & 4,14 & 4,20 & 4,18 & 4,19 & 4,23 \\
\hline Costa Rica & 4,20 & 4,25 & 4,23 & 4,31 & 4,27 & 4,34 & 4,35 & 4,42 \\
\hline Ecuador & 3,36 & 3,56 & 3,58 & 3,65 & 3,82 & 3,94 & 4,18 & 4,22 \\
\hline El Salvador & 4,00 & 4,02 & 3,99 & 3,89 & 3,99 & 3,80 & 3,84 & 4,01 \\
\hline Guatemala & 3,92 & 3,96 & 4,04 & 3,94 & 4,00 & 4,01 & 4,04 & 4,10 \\
\hline
\end{tabular}


Cuadro 4 (conclusión)

\begin{tabular}{lllllllll}
\hline & 2008 & 2009 & 2010 & 2011 & 2012 & 2013 & 2014 & 2015 \\
\hline México & 4,18 & 4,19 & 4,19 & 4,29 & 4,23 & 4,34 & 4,36 & 4,27 \\
\hline Nicaragua & 3,32 & 3,44 & 3,57 & 3,41 & 3,61 & 3,73 & 3,84 & 3,82 \\
\hline Panamá & 4,18 & 4,21 & 4,24 & 4,33 & 4,35 & 4,49 & 4,50 & 4,43 \\
\hline Perú & 3,93 & 4,01 & 3,95 & 4,11 & 4,21 & 4,28 & 4,25 & 4,24 \\
\hline
\end{tabular}

Fuente: Elaboración propia, sobre la base de datos de K. Schwab (ed.), The Global Competitiveness Report 2016-2017, Ginebra, 2016 [en línea] http://www3.weforum.org/docs/GCR2016-2017/05FullReport/TheGlobalCompetitiveness Report2016-2017_FINAL.pdf.

\section{e) Tratados comerciales}

Como se señala, el comercio ha sido un factor determinante del crecimiento del conjunto de países objeto de estudio. Si bien hubo un importante incremento del comercio en el pasado 2009-2011 gracias al aumento de los precios de las materias primas (CEPAL, 2014), en la actualidad el comercio internacional presenta un estancamiento, principalmente debido a la reducción de la demanda exterior de materias primas por parte de China. No obstante, existe un impulso de crecimiento que se ve favorecido por el comercio Sur-Sur, promovido por la cuarta revolución industrial (macrodatos y comercio electrónico) (UNCTAD, s/fa).

Debido a la enorme importancia del comercio en las economías analizadas, es necesario estudiar la apertura comercial que han llevado a cabo estos países, con el objetivo común de liberalizar el intercambio mercantil y eliminar barreras, para favorecer el comercio con países tanto de la región como de fuera de ella. Es necesario, por tanto, realizar un análisis descriptivo de la política comercial en lo referente a tratados de libre comercio de los países considerados en esta investigación.

América Latina y el Caribe ha promovido una multitud de acuerdos regionales, con la finalidad de ampliar el mercado de bienes y servicios, eliminando barreras entre los países vecinos. Sin embargo, estos proyectos - como la Comunidad Andina (CAN), creada en 1969 con el nombre de Pacto Andino, la Organización del Tratado de Cooperación Amazónica (OTCA), creada en 1978, y la Asociación Latinoamericana de Integración (ALADI), creada en 1980 - no han alcanzado los resultados esperados. Los mecanismos de integración comercial activos más importantes en la actualidad son el MERCOSUR, creado en 1991, la Alianza Bolivariana para los Pueblos de Nuestra América-Tratado de Comercio de los Pueblos (ALBA-TCP), establecida en 2004, y la Alianza del Pacífico (AP), creada en 2011.

Mediante estos acuerdos se busca la creación de bloques económicos que favorezcan las relaciones comerciales y que ayuden al desarrollo de los países integrantes de cada uno. No obstante, las posturas difieren ampliamente a la hora de tomar decisiones, pues van desde el aperturismo de la Alianza del Pacífico hasta el proteccionismo de la ALBA-TCP.

Los países integrantes de la Alianza del Pacífico (Chile, Colombia, México y Perú) presentan un modelo claro de apertura y liberalización. Se trata de Estados que han ratificado a la vez tratados de libre comercio con diversas economías de fuera de la región. Estos tratados son herramientas de política comercial que ayudan a desarrollar un intercambio de exportaciones e importaciones competitivo, generando, además, mejoras económicas, laborales y sociales. En muchas ocasiones, provocan el aumento de la inversión extranjera ya que otorgan seguridad y estabilidad a los inversionistas.

Al analizar los tratados de libre comercio que han ratificado estos países se observa que en los últimos años los que han provocado un mayor impacto en las economías son los firmados con los países asiáticos. En conclusión, se podría afirmar que:

- Los países latinoamericanos han establecido una multitud de acuerdos regionales. Muchos de ellos no han prosperado, mientras que otros han favorecido el comercio entre los países 
integrantes (MERCOSUR). En ese marco, la Alianza del Pacífico surge como un bloque comercial que impulsa los intercambios entre los países integrantes y también hacia fuera de la región.

- La mayoría de los tratados de libre comercio que han ratificado los países latinoamericanos se han establecido con países de la misma región, con asociados europeos y con los Estados Unidos. No obstante, durante el período analizado, las economías asiáticas, específicamente China y el Japón, son las que en mayor medida mejoran sus posiciones en la lista de los principales socios comerciales de esas economías.

\section{Modelo econométrico}

La muestra utilizada se compone de 23 puertos de la costa oeste de América Latina y 8 observaciones temporales (2008 a 2015). Los puertos son: Antofagasta, Arica, Coronel, lquique, Lirquén, Mejillones, San Antonio, San Vicente y Valparaíso (Chile); Buenaventura (Colombia); Caldera (Costa Rica); Esmeraldas y Guayaquil (Ecuador); Acajutla (El Salvador); Puerto Quetzal (Guatemala); Ensenada, Lázaro Cárdenas y Manzanillo (México); Corinto (Nicaragua); Balboa (Panamá); Callao, Matanari y Paita (Perú). Como se dispone de más unidades de datos transversales (23 puertos) que de series temporales (8 años), se emplean las estimaciones con datos de panel $(N>T)$, en lugar de datos de series temporales $(N<T)$.

Greene (2003) afirma que la ventaja fundamental de los datos de panel, en relación con los datos de corte transversal, es que permiten una mayor flexibilidad en los modelos, considerando las diferencias entre las unidades que lo componen. Entre las principales ventajas cabe destacar:

- No se necesitan supuestos rígidos para datos de panel, mientras que en el caso de datos transversales, especialmente para el modelo de máxima verosimilitud (MLE), los supuestos de distribución de cada componente de errores son necesarios para separar la ineficiencia técnica del ruido estadístico; además, el análisis MLE requiere que la ineficiencia técnica sea independiente de los regresores.

- La eficiencia técnica para cada unidad puede ser estimada de forma consistente con datos de panel; aunque también es posible realizar la estimación con datos transversales, esta no es consistente.

- Los datos de panel proporcionan más información sobre el comportamiento de las unidades a lo largo del tiempo, que no puede ser analizado con datos transversales, como, por ejemplo, cambios de estructura y factores variantes o invariantes en el tiempo a través de análisis de efectos fijos y aleatorios.

La especificación del modelo parte de la estructura básica propuesta por Greene (2003):

$$
Y_{i t}=x_{i t}^{\prime} \beta+z_{i}^{\prime} \alpha+\varepsilon_{i t}
$$

Donde:

$Y_{i t}$ : es la variable que se busca explicar;

$x_{i t}^{\prime}: \quad$ es un vector de variables explicativas $(K \times 1)$;

$z_{i}^{\prime} \alpha$ : son los efectos individuales, donde $z_{i}$ contiene un término constante y una serie de variables individuales o grupales, que pueden ser observables o no observables;

$\beta$ : $\quad$ es el vector de pendientes de la ecuación;

$t: \quad$ se refiere a la serie de tiempo que llega hasta el período $T(t=1,2, \ldots, T)$;

$i: \quad$ se refiere a los individuos, siendo el último el individuo $N(i=1,2, \ldots, N)$;

$\varepsilon_{i t}: \quad$ es el término de error. 
A partir de la estructura del modelo econométrico planteado se presenta la siguiente especificación:

$$
L P I B p c_{i t}=\beta_{0}+\beta_{1} \cdot L T E U_{i t}+\beta_{2} \cdot L D e s_{i t}+\beta_{3} \cdot L G C I_{i t}+\beta_{4} \cdot T_{i t}+\varepsilon_{i t}
$$

Donde:

$\operatorname{LPIBPc}_{i t}$ : logaritmo natural del PIB per cápita en paridad del poder adquisitivo expresado en dólares constantes de 2011 del país donde se sitúa el puerto i-ésimo en el año t-ésimo (Banco Mundial, s/f);

$L_{T E U_{i t}}$ : logaritmo natural de los TEU manipulados en el puerto i-ésimo en el año t-ésimo (CEPAL, s/f);

LDes $_{i t}$ : logaritmo natural de la tasa de desempleo del país donde se sitúa el puerto i-ésimo en el año t-ésimo (Banco Mundial, s/f);

$L G C I_{i t}$ : logaritmo natural del índice de competitividad del país donde se sitúa el puerto i-ésimo en el año t-ésimo (Schwab, 2016);

$T_{i t}$ : $\quad$ variable ficticia (dummy) que toma valor 1 en el año y país que firma un acuerdo comercial de carácter aperturista (Alianza del Pacífico) o que ratifica un tratado de libre comercio con algún país asiático en un año dado y 0 en caso contrario, en el puerto i-ésimo en el año t-ésimo;

$\beta_{m}: \quad$ representa cada uno de los coeficientes de las $m$ variables explicativas;

t: $\quad$ de 2008 a 2015;

$i$ : $\quad$ cada uno de los puertos analizados;

$\varepsilon_{i t}$ : $\quad$ es el término de error.

El modelo de efectos fijos es el modelo más utilizado en economía y ciencias políticas (Schurer y Yong, 2012). Además, según Wooldridge (2010) la transformación de efectos fijos es útil para el análisis de políticas y la evaluación de programas económicos. Brüderl y Ludwig (2015) sostienen que los modelos de regresión estándar proporcionan estimaciones sesgadas si existen características intrínsecas no observadas, mientras que la estimación de efectos fijos es un método que puede proporcionar estimaciones no sesgadas en estas circunstancias.

Antes de la estimación mediante un modelo de efectos fijos (Brüderl y Ludwig, 2015; Gangl, 2010; Allison, 2009), efectos aleatorios o errores estándar corregidos para panel (panel-corrected standard errors (PCSE)) (Rodríguez, 2017; Marques y Fuinhas, 2012; Reed y Webb, 2010), es necesario realizar los test estadísticos para conocer las características de los datos que forman la muestra y decidir correctamente tanto la especificación como el método econométrico adecuado.

A través de los test se comprobará la existencia de heterocedasticidad, autocorrelación temporal y/o correlación contemporánea entre unidades transversales.

Además, si se detecta presencia de heterocedasticidad (test de Wald modificado para heterocedasticidad grupal), esto se traduce en que la estimación inicial de efectos fijos no sea precisa y, por ello, se realiza una estimación de efectos fijos con un estimador para la varianza de los errores robusta a heterocedasticidad.

Por último, la presencia de autocorrelación y correlación contemporánea provoca que se utilice finalmente el método de estimación de errores estándar corregidos para datos de panel enunciado por Beck y Katz (1995). Se emplea para el cálculo de los errores estándar y las estimaciones de la varianza-covarianza; se asume que los errores son por defecto heterocedásticos y tienen correlación contemporánea entre los paneles (entidades).

Las variables del modelo se expresan en logaritmos, con la excepción de la variable referente a tratados, que se incorpora como una variable ficticia (dummy). 


\section{a) Test de Hausman}

La prueba estadística propuesta por Hausman (1978) está basada en un test chi-cuadrado cuyo objetivo es determinar si entre dos estimaciones dadas existen diferencias sistemáticas y significativas. El test de Hausman ha sido ampliamente empleado para determinar la conveniencia de utilizar un estimador de efectos fijos o de efectos aleatorios en el contexto de especificaciones con paneles de datos (Wooldrige, 2010; Greene, 2001). Las especificaciones con efectos fijos son, en principio, más apropiadas cuando se emplea un conjunto de observaciones divididas en unidades transversales, como ocurre con los paneles de datos. Sin embargo, de acuerdo con Baltagi (2008), si el número de unidades transversales es demasiado alto en relación con el número de unidades temporales, la estimación de un modelo de efectos fijos causará una pérdida significativa de grados de libertad, lo que implicará generalmente una varianza mayor de los parámetros estimados; en ese caso, se sugiere la utilización del estimador de efectos aleatorios.

En el cuadro 5 se presentan los resultados del test de Hausman para determinar la conveniencia o no de emplear un modelo de efectos aleatorios.

\section{Cuadro 5}

Resultados del test de Hausman

\begin{tabular}{lcccc}
\hline & Coeficientes fijos $(\mathrm{b})$ & Coeficientes aleatorios (B) & Diferencia $(\mathrm{b}-\mathrm{B})$ & Error estándar \\
\hline LTEU & 0,0298407 & 0,0303932 & $-0,0005525$ & 0,0029095 \\
\hline LDes & $-0,2317544$ & $-0,1801522$ & $-0,0516022$ & 0,0077272 \\
\hline LGCl & 0,521414 & 0,8465897 & $-0,3251758$ & 0,0417249 \\
\hline Tratados & 0,0640417 & 0,1101613 & $-0,0461196$ & 0,0072828 \\
\hline \multicolumn{5}{c}{ Test: $H_{0}:$ Diferencia en coeficientes no sistemática } \\
\hline \multicolumn{5}{c}{$\chi^{2}(7)=(b-B) '\left[\left(V \_b-V \_B\right) \wedge(-1)\right](b-B)=64,28$} \\
\hline
\end{tabular}

Fuente: Elaboración propia.

Nota: Cálculos realizados en STATA 13. LTEU: logaritmo natural de los TEU (unidad equivalente a 20 pies) manipulados; LDes: logaritmo natural de la tasa de desempleo; LGCl: logaritmo natural del índice de competitividad.

Los resultados obtenidos permiten rechazar la hipótesis nula $(p<0,05)$ de igualdad al $95 \%$ de confianza y, por lo tanto, se debe asumir como adecuada la estimación de efectos fijos.

\section{b) Test de Wooldridge para autocorrelación en datos de panel}

La autocorrelación en una serie temporal de un proceso $X_{t}$ es la correlación de dicho proceso con una versión desplazada en el tiempo de la propia serie temporal. Según Drukker (2003), la autocorrelación en modelos con datos de panel causa sesgos en los errores estándar y lleva a resultados menos eficientes, siendo necesario identificar la autocorrelación en términos de error idiosincrásico. Baltagi y Wu (1999) enunciaron un test similar al de Wooldridge (2003) cuyo funcionamiento se mostró óptimo con una serie de supuestos referentes a los efectos individuales del modelo que se buscaba probar. Sin embargo, según Drukker (2003) el test de Wooldridge puede ser menos potente que otros test altamente parametrizados, pero resulta más robusto, es decir, requiere de relativamente pocos supuestos y es fácil de implementar.

Bajo la hipótesis nula de no correlación serial, los residuos de la regresión de las primeras diferencias deberían tener una autocorrelación de -0,5. Esto implica que el coeficiente de una regresión basada en los residuos retardados sobre los residuos corrientes debe ser -0,5. Los resultados de este test se muestran en el cuadro 6. 
Cuadro 6

Resultados del test de Wooldridge

\begin{tabular}{c}
\hline$H_{0}$ : No autocorrelación de primer orden \\
$F(1,22)=133,025$ \\
Prob $>F=0,0000$
\end{tabular}

Fuente: Elaboración propia.

Nota: Cálculos realizados en STATA 13.

La hipótesis nula de no correlación de primer orden se rechaza y se asume la existencia de autocorrelación de primer orden en el modelo.

\section{c) Test para homocedasticidad grupal}

Para poder utilizar una inferencia basada en mínimos cuadrados ordinarios (Ordinary Least Squares (OLS)) de la matriz de covarianzas es necesario asumir, además de no autocorrelación, homocedasticidad en los datos. La diferencia en la varianza de cada una de las entidades (o paneles) del modelo habrá de probarse para aceptar o rechazar la hipótesis de homocedasticidad.

En el cuadro 7 se ejecuta el test de Wald modificado para probar la existencia de heterocedasticidad grupal en los residuos de la regresión del modelo específico con efectos fijos tal como propone Greene (2003). Se prueba la hipótesis nula $\sigma^{2}{ }_{i}=\sigma^{2}$ para $i=1,2, \ldots, N_{g}$, donde $N_{g}$ es el número de unidades transversales. El resultado de este test de Wald modificado se distribuirá como una $\chi^{2}\left[N_{g}\right]$ bajo la hipótesis nula de homocedasticidad. Según Baum (2001), las propiedades de este test no son muy potentes para muestras pequeñas cuando $\mathrm{N}>\mathrm{T}$, por lo que es conveniente tomar con cautela los resultados obtenidos.

\section{Cuadro 7}

Test de Wald modificado para heterocedasticidad grupal

\begin{tabular}{c}
\hline $\mathrm{H}_{0}: \sigma^{2}{ }_{i}=\sigma^{2}$, para todo $i=1,2,3, \ldots, 34$ \\
$\chi^{2}(23)=1374,93$ \\
$\operatorname{Prob}>\chi^{2}=0,0000$
\end{tabular}

Fuente: Elaboración propia.

Nota: Cálculos realizados en STATA 13.

Se rechaza la hipótesis nula de homocedasticidad grupal en los residuos de la regresión.

\section{d) Test de Pesaran para dependencia entre entidades}

Según De Hoyos y Sarafidis (2006), los modelos con datos de panel tienden a presentar niveles considerables de dependencia entre entidades transversales, correlación contemporánea, que podría aparecer debido a la presencia de choques comunes a dichas entidades y/o componentes no observados que formarían parte del término de error. Esta afirmación está recogida en trabajos como los de Robertson y Symons (2000), Anselin (2001), Pesaran (2004) y Baltagi (2008).

El test de Pesaran (véase el cuadro 8) prueba la hipótesis de independencia entre entidades transversales en un modelo de datos de panel con $\mathrm{N}>\mathrm{T}$, a través del proceso paramétrico propuesto por Pesaran (2004). Este hecho es el que ha llevado a excluir la posibilidad de emplear el test de Breusch-Pagan para la detección de correlación contemporánea en los residuos del modelo, en beneficio de la alternativa de Pesaran. 


\section{Cuadro 8}

Test de Pesaran

\begin{tabular}{c}
$\mathrm{H}_{0}$ : Independencia entre entidades transversales \\
\hline Test de Pesaran para dependencia de entidades $=18,156, \mathrm{Pr}=0,0000$ \\
\hline Valor absoluto medio de los valores fuera de la diagonal $=0,594$ \\
\hline
\end{tabular}

Fuente: Elaboración propia.

Nota: Cálculos realizados en STATA 13.

Se rechaza la hipótesis nula de independencia entre las entidades transversales y, por tanto, se asume la existencia de dependencia entre entidades transversales (correlación contemporánea).

\section{Resultados}

Mediante los test realizados se ha detectado que la muestra presenta las siguientes características: heterocedasticidad, autocorrelación y correlación contemporánea. Además, el test de Hausman indica que entre una estimación de efectos fijos y otra de efectos aleatorios, es preferible la de efectos fijos (Wooldridge, 2010; Greene, 2001).

En la investigación realizada por Rodríguez (2017) se presenta una muestra de datos con las mismas características y se realizan determinadas estimaciones; se concluye que la estimación con errores estándar corregidos para panel es la más adecuada en estas circunstancias. Finalmente, en esta investigación la estimación que ofrece mejores resultados es el modelo PCSE mediante la transformación previa de Prais y Winsten (1954) para la corrección de la autocorrelación de primer orden. También existe la posibilidad de calcular un coeficiente autorregresivo para cada panel (lo que en la literatura se conoce como panel-specific AR1) o calcular un coeficiente autorregresivo común a todos los paneles. A priori, al tratarse de una muestra compuesta por un conjunto de países heterogéneos, aunque casi todos en desarrollo (con la excepción de Chile y México), se podría asumir la existencia de un coeficiente autorregresivo diferente para cada puerto. Sin embargo, siguiendo a Beck y Katz (1995) se recomienda, al igual que en los modelos de series de tiempo de corte transversal y de panel en los que los parámetros de interés $\beta$ no varían para cada unidad transversal, que el parámetro de autocorrelación tampoco debería hacerlo.

Dado que el panel de datos del que se compone la muestra es equilibrado, la estrategia que se ha seguido para el cálculo de las covarianzas se basa en emplear todas las observaciones comunes a cada pareja de paneles para el cálculo de cada elemento de la matriz de covarianzas. Como método para calcular el parámetro de autocorrelación se ha elegido el método de cálculo de la autocorrelación de series temporales, siendo el resto de las alternativas consistentes y asintóticamente equivalentes.

En el cuadro 9 se presentan los resultados de las tres estimaciones: efectos fijos básicos, es decir, el estimador de efectos fijos sin ningún tipo de corrección; efectos fijos con un estimador para la varianza de los errores robusta a heterocedasticidad y, por último, el modelo PCSE con las características propias de la muestra, es decir, incluidas correcciones para autocorrelación, correlación contemporánea y heterocedasticidad. La estimación se realiza para los 23 puertos durante 8 años, lo que forma un total de 184 observaciones. Todas las variables del modelo están expresadas en forma logarítmica, excepto la variable referente a tratados, que se trata como una variable ficticia (dummy). 
Cuadro 9

Modelo de efectos fijos y con errores estándar corregidos para panel (PCSE)

\begin{tabular}{|c|c|c|c|c|c|}
\hline \multicolumn{6}{|c|}{$L P I B p c_{i t}=\beta_{0}+\beta_{1} \cdot L T E U_{i t}+\beta_{2} \cdot L D e s_{i t}+\beta_{3} \cdot L G C I_{i t}+\beta_{4} \cdot T_{i t}+\varepsilon_{i t}$} \\
\hline & & & Efectos fijos & $\begin{array}{c}\text { Efectos fijos } \\
\text { (robust) }\end{array}$ & PCSE \\
\hline$\beta_{0}$ & & & 8,982853 & 8,982853 & 5,400565 \\
\hline Error estándar & & & 0,2009241 & 0,4333786 & 0,6216013 \\
\hline$P>|t|$ & & & 0,000 & 0,000 & 0,000 \\
\hline \multirow{3}{*}{ LTEU } & & $\beta_{1}$ & 0,0298407 & 0,0298407 & 0,0155947 \\
\hline & & Error estándar & 0,0083282 & 0,0130366 & 0,0074627 \\
\hline & & $P>|t|$ & 0,000 & 0,032 & 0,037 \\
\hline \multirow{3}{*}{ LDes } & & $\beta_{2}$ & $-0,2317544$ & $-0,2317544$ & 0,0383143 \\
\hline & & Error estándar & 0,0241899 & 0,059507 & 0,0508677 \\
\hline & & $P>|t|$ & 0,000 & 0,001 & 0,451 \\
\hline \multirow{3}{*}{ LGCl } & & $\beta_{3}$ & 0,521414 & 0,521414 & 2,612246 \\
\hline & & Error estándar & 0,1267077 & 0,2265887 & 0,4352533 \\
\hline & & $P>|t|$ & 0,000 & 0,031 & 0,000 \\
\hline \multirow{3}{*}{$\mathrm{T}$} & & $\beta_{4}$ & 0,0640417 & 0,0640417 & 0,2836674 \\
\hline & & Error estándar & 0,0231973 & 0,0117699 & 0,0508644 \\
\hline & & $P>|t|$ & 0,006 & 0,000 & 0,000 \\
\hline N & & & 184 & 184 & 184 \\
\hline $\mathrm{F}$ & & & 55,74 & 538,78 & \\
\hline$P>F$ & & & 0,000 & 0,000 & \\
\hline Wald $\mathrm{Chi}^{2}$ & & & & & 164,79 \\
\hline $\mathrm{P}>\mathrm{Chi}^{2}$ & & & & & 0,000 \\
\hline $\operatorname{COV}\left(v_{j}, x_{i, j}\right)$ & & & 0,2739 & 0,2739 & \\
\hline Rho & & & 0,98535966 & 0,98535966 & 0,7022694 \\
\hline $\mathrm{R}^{2}$ & & & & & 0,9955 \\
\hline \multirow{3}{*}{$\mathrm{R}^{2}$} & Within & & 0,5868 & 0,5868 & \\
\hline & Between & & 0,2488 & 0,2488 & \\
\hline & Overall & & 0,2346 & 0,2346 & \\
\hline Covarianzas estimadas & & & & & 276 \\
\hline Autocorrelaciones estimadas & & & & & 1 \\
\hline Coeficientes estimados & & & & & 5 \\
\hline
\end{tabular}

Fuente: Elaboración propia.

Nota: Cálculos realizados en STATA 13.

Los datos obtenidos al estimar los modelos permiten observar algunas diferencias significativas. En cuanto a los resultados en el valor de Rho, en las dos estimaciones de efectos fijos Rho toma el valor de 0,98535966, mientras que en el caso de PCSE el valor se reduce a 0,7022694. Las estimaciones de efectos fijos son las que presentan un mayor valor de Rho; sin embargo, si se atiende al $\mathrm{R}^{2}$ within $(0,5868)$, between $(0,2488)$ y overall $(0,2346)$, se observa que los valores son mucho más bajos que el $R^{2}(0,9955)$ del modelo PCSE.

En las estimaciones de efectos fijos, las variables analizadas (LTEU, LDes, LGCl y tratados) se muestran significativas con un nivel de confianza del 95\% y los coeficientes estimados no presentan variaciones. Los resultados muestran que un incremento del $1 \%$ en el indicador de TEU supone un crecimiento del 0,0298\% del PIB per cápita. El aumento del 1\% de la tasa de desempleo se traduce 
en una reducción del PIB per cápita de un 0,2318\%. En cuanto al índice de competitividad, se observa que una mejora de un 1\% redunda en un aumento del PIB per cápita del 0,5214\% y, finalmente, la existencia de una política comercial de carácter aperturista supone un incremento del PIB per cápita de un $0,0640 \%$.

Las estimaciones de efectos fijos son consistentes, pero no eficientes, dadas las características de las variables que componen la muestra. Por ello, se realiza la estimación con errores estándar corregidos para panel, donde la variable LDes es la única que no se muestra significativa; esto puede deberse - como se recoge en la investigación de Cipoletta Tomassian, Pérez y Sánchez (2010) - a que las inversiones realizadas en infraestructuras en el área geográfica analizada han sido bajas y, por ello, no han llegado a influir en el mercado laboral.

En lo referente a la estimación del modelo PCSE, las variables LTEU, LGCl y tratados, además de mostrarse significativas, presentan signos positivos. En términos cuantitativos el incremento del $1 \%$ del índice de TEU supone un crecimiento del 0,0156\% del PIB per cápita. Un incremento del índice de competitividad del 1\% supone una variación del 2,61\% del PIB per cápita. Además, la existencia de una política económica basada en acuerdos de libre comercio de carácter aperturista o tratados con países asiáticos supone un aumento del PIB per cápita del 0,2837\%.

Los resultados muestran que el índice de competitividad tiene un efecto importante en el PIB per cápita. Este porcentaje se justifica, sobre todo, debido a la configuración de la variable, ya que el índice se elabora a través de un análisis de diferentes aspectos de cada país y los valores oscilan entre 1 y 7 . En concreto, en los diez países analizados varía entre 3,32 y 4,75 en el período estudiado (2008 a 2015). En resumen, su escasa variación y la agrupación de tantos aspectos en un indicador se traduce en que un leve incremento del índice suponga una mejora considerable en el país y, por tanto, que este efecto se materialice de forma significativa en el PIB per cápita.

Finalmente, la existencia de acuerdos comerciales de carácter aperturista o de tratados con países asiáticos significa que la política comercial aperturista que se llevó adelante a finales del siglo XX en los países latinoamericanos favoreció el desarrollo de estas economías y, además, propició que estas zonas geográficas no sufrieran tan duramente los efectos de la crisis financiera mundial.

\section{Conclusiones}

El transporte marítimo representa el 80\% del comercio mundial (UNCTAD, s/fb). Esta investigación se centra en una parte del transporte marítimo, aquella en que la mercancía se transporta en contenedores, sobre todo, por dos importantes razones: en primer lugar, por el auge que exhibe el comercio en la logística de cajones con medidas estándar (TEU) y, en segundo lugar, porque la mercancía en contenedores constituye el comercio con mayor valor agregado en el movimiento de mercancías. En definitiva, este tipo de transporte es una imagen fidedigna del comercio de bienes con un mayor valor agregado en los países y, por tanto, sus efectos son muy significativos para las economías, ya que favorecen un importante desarrollo socioeconómico.

La estimación realizada arroja resultados que respaldan la hipótesis de que el aumento del comercio de mercancía en contenedores a través de los puertos repercute en el crecimiento de la economía del país y, por tanto, que este tipo de comercio redunda en beneficios para toda la población. En concreto, en lo referente a la estimación, el índice de TEU, el índice de competitividad, así como la variable referente a los tratados comerciales, además de mostrarse significativas presentan signos positivos y, por tanto, sus efectos en el PIB per cápita son positivos. En términos cuantitativos, el incremento del 1\% del índice de TEU supone un crecimiento del 0,0156\% del PIB per cápita, en tanto que un incremento del 1\% del índice de competitividad supone una variación del 2,61\% del PIB per cápita. 
Mediante la variable denominada "tratados" se pretende medir el efecto de una política de carácter aperturista y potenciada en el período analizado por el comercio con los países asiáticos, con los que diversas economías de la región han suscrito tratados de libre comercio. La existencia de esta políica supone un aumento del PIB per cápita del 0,2837\%.

Los resultados muestran que el índice de competitividad es la variable que mayor efecto presenta sobre el PIB per cápita. Esto se puede explicar por la forma de obtención del índice y por la escasa variabilidad que presenta en la muestra analizada (en la que toma valores entre 3,32 y 4,75); por ello, un leve incremento del índice se traduce en un gran impacto en el PIB per cápita.

Finalmente, en el período analizado, comprendido entre 2008 y 2015, las economías de los países de la costa oeste de América Latina se han visto favorecidos por los instrumentos de política comercial que han desarrollado y por el alto precio de las materias primas, cuyos efectos se pueden medir a través de la mercancía transportada en contenedores, que es un fiel estimador del comercio. No obstante, cabe concluir que estos países necesitan reforzar las inversiones en infraestructuras portuarias, modernizándolas y adaptándolas a las necesidades actuales, ya que estas instalaciones generan beneficios económicos en el largo plazo para los países, y por tanto, para la población.

\section{Bibliografía}

Allison, P. D. (2009), Fixed Effects Regression Models, Nueva York, Sage.

Anselin, L. (2001), "Spatial effects in econometric practice in environmental and resource economics", American Journal of Agricultural Economics, vol. 83, № 3, Oxford, Oxford University Press.

Baltagi, B. (2008), Econometric Analysis of Panel Data, Hoboken, Wiley.

Baltagi, B. H. y P. X. Wu (1999), "Unequally spaced panel data regressions with AR (1) disturbances", Econometric Theory, vol. 15, № 6, Nueva York, Cambridge University Press.

Banco Mundial (s/f), "Indicators" [en línea] https://data.worldbank.org/indicator?tab=all.

Baum, C. F. (2001), "XTTEST3: Stata module to compute Modified Wald statistic for groupwise heteroskedasticity", Statistical Software Components, № S414801, Boston, Boston College.

Beck, N. y J. N. Katz (1995), "What to do (and not to do) with time-series cross-section data", American Political Science Review, vol. 89, № 3, Nueva York, Cambridge University Press.

Bernal-Meza, R. (2015), "Alianza del Pacífico versus Alba y Mercosur: entre el desafío de la convergencia y el riesgo de la fragmentación de Sudamérica", Pesquisa e Debate, vol. 26, № 1, São Paulo, Pontificia Universidad Católica de São Paulo.

Bernhofen, D. M., Z. El-Sahli y R. Kneller (2016), "Estimating the effects of the container revolution on world trade", Journal of International Economics, vol. 98, Amsterdam, Elsevier.

Bitar, S. (2016), "Las tendencias mundiales y el futuro de América Latina", serie Gestión Pública, № 78 (LC/L.3681), Santiago, Comisión Económica para América Latina y el Caribe.

Bolaky, B. y C. L. Freund (2004), "Trade, regulations, and growth", Policy Research Working Paper, № 3255, Washington, D.C., Banco Mundial.

Brüderl, J. V. y V. Ludwig (2015), "Fixed-effects panel regression", The Sage Handbook of Regression Analysis and Causal Inference, H. Best y C. Wolf (eds.), Los Angeles, Sage.

CEPAL (Comisión Económica para América Latina y el Caribe) (2014), Balance Preliminar de las Economías de América Latina y el Caribe, 2014 (LC/G.2632-P), Santiago. (s/f), "Perfil marítimo y logístico de América Latina y el Caribe" [en línea] http://bit.ly/2fV5bzS.

Chang, V. y B. Tovar (2014), "Drivers explaining the inefficiency of Peruvian and Chilean ports terminals", Transportation Research Part E: Logistics and Transportation Review, vol. 67, Amsterdam, Elsevier.

Cipoletta Tomassian, G., G. Pérez y R. Sánchez (2010), "Políticas integradas de infraestructura, transporte y logística: experiencias internacionales y propuestas iniciales", serie Recursos Naturales e Infraestructura, № 150 (LC/L.3226-P), Santiago, Comisión Económica para América Latina y el Caribe (CEPAL).

Corbett, J. J. y J. Winebrake (2008), The Impacts of Globalisation on International Maritime Transport Activity: Past Trends and Future Perspectives, París, Organización de Cooperación y Desarrollo Económicos (OECD)/Foro Internacional de Transporte (ITF). 
Coto-Millán, P., J. Baños-Pino y A. Rodríguez-Álvarez (2000), "Economic efficiency in Spanish ports: some empirical evidence", Maritime Policy \& Management, vol. 27, № 2, Abingdon, Taylor \& Francis.

De Hoyos, R. E. y V. Sarafidis (2006), "Testing for cross-sectional dependence in panel-data models", The Stata Journal, vol. 6, № 4, Nueva York, Sage.

Drukker, D. M. (2003), "Testing for serial correlation in linear panel-data models", The Stata Journal, vol. 3, № 2, Nueva York, Sage.

Fay, M. y M. Morrison (2007), Infrastructure in Latin America and the Caribbean: Recent Developments and Key Challenges, Washington, D.C., Banco Mundial.

Gangl, M. (2010), "Causal inference in sociological research", Annual Review of Sociology, vol. 36, Palo Alto, Annual Reviews.

Girón, A. (2015), "Zonas de Libre Comercio. ¿Un camino para el desarrollo?”, Problemas del Desarrollo, vol. 46, № 180, Amsterdam, Elsevier.

Greene, W. (2003), Econometric Analysis, Nueva Jersey, Prentice Hall. (2001), "Estimating econometric models with fixed effects", Leonard N. Stern School of Business Working Paper, N 44, Nueva York, Universidad de Nueva York.

Grossman, G. M. y E. Helpman (1993), Innovation and Growth in the Global Economy, Cambridge, MIT Press.

Grossmann, H. y otros (2007), "Growth potential for maritime trade and ports in Europe", Intereconomics, vol. 42, № 4, Amsterdam, Elsevier.

Guisan, M. C. (2008), "Rates, ratios and per capita variables in international models: analysis of investment and foreign trade in OECD countries", International Journal of Applied Econometrics and Quantitative Studies, vol. 5, № 2, Santiago de Compostela, Universidad de Santiago de Compostela.

Hausman, J. A. (1978), "Specification tests in econometrics", Econometrica, vol. 46, № 6, Nueva York, Sociedad Econométrica.

López-Bermúdez, B., M. J. Freire y C. Pais (2018), "Crecimiento económico y transporte marítimo en América Latina, 2000-2015: los efectos de políticas comerciales y modelización con datos de panel”, Estudios Económicos Regionales y Sectoriales, vol. 18, № 1, Santiago de Compostela, Universidad de Santiago de Compostela.

Marques, A. C. y J. A. Fuinhas (2012), "Are public policies towards renewables successful? Evidence from European countries", Renewable Energy, vol. 44, Amsterdam, Elsevier.

Micco, A. y N. Pérez (2001), "Maritime transport costs and port efficiency", ponencia presentada en el seminario Towards Competitiveness: The Institutional Path, Banco Interamericano de Desarrollo (BID), Santiago, 16 de marzo [en línea] http://citeseerx.ist.psu.edu/viewdoc/download?doi=10.1.1.194.6075 \&rep=rep1\&type=pdf.

Núñez-Sánchez, R. y P. Coto-Millán (2012), "The impact of public reforms on the productivity of Spanish ports: a parametric distance function approach", Transport Policy, vol. 24, Amsterdam, Elsevier.

Pesaran, M. H. (2004), "General diagnostic tests for cross section dependence in panels", CESifo Working Paper, № 1229, Múnich, Grupo CSEifo.

Prais, S. J. y C. B. Winsten (1954), "Trend estimators and serial correlation", Cowles Commission Discussion Paper, №383, Chicago, Universidad de Chicago.

Radelet, S. y J. Sachs (1998), "Shipping costs, manufactured exports, and economic growth", ponencia presentada en la Reunión Anual de la Asociación Estadounidense de Economía, Chicago, 3-5 enero.

Ramos-Real, F. J. y B. Tovar (2010), "Productivity change and economies of scale in container port terminals: a cost function approach", Journal of Transport Economics and Policy, vol. 44, № 2, Universidad de Bath.

Redding, S. (2002), "Specialization dynamics", Journal of International Economics, vol. 58, № 2, Amsterdam, Elsevier.

Reed, W. R. y R. Webb (2010), "The PCSE estimator is good-just not as good as you think", Journal of Time Series Econometrics, vol 2, № 1, Berlín, De Gruyter.

Rivera-Batiz, L. A. y P. M. Romer (1991), "International trade with endogenous technological change", European Economic Review, vol. 35, № 4, Amsterdam, Elsevier.

Robertson, D. y J. Symons (2000), "Factor residuals in SUR regressions: estimating panels allowing for cross sectional correlation", CEP Discussion Papers, № CEPDP0473, Londres, Escuela de Economía y Ciencia Política de Londres.

Rodrigue, J. P., C. Comtois y B. Slack (2013), The Geography of Transport Systems, Nueva York, Routledge.

Rodrigue, J. P. y otros (2010), "Functions and actors of inland ports: European and North American dynamics", Journal of Transport Geography, vol. 18, № 4, Amsterdam, Elsevier. 
Rodríguez, M. (2017), "El efecto del gasto público sanitario y educativo en la determinación del bienestar de los países de la OCDE", tesis de doctorado, La Coruña, Facultad de Economía y Empresa, Universidad de La Coruña.

Roll, Y. y Y. Hayuth (1993), "Port performance comparison applying data envelopment analysis (DEA)", Maritime Policy and Management, vol. 20, № 2, Abingdon, Taylor \& Francis.

Romer, P. M. (1990), "Endogenous technological change", Journal of Political Economy, vol. 98, № 5, Chicago, The University of Chicago Press.

Sánchez, R. (2004), "Puertos y transporte marítimo en América Latina y el Caribe: un análisis de su desempeño reciente", serie Recursos Naturales e Infraestructura, Nº 82 (LC/L.2227-P), Santiago, Comisión Económica para América Latina y el Caribe (CEPAL).

Sánchez, R. y otros (2015), "Transporte marítimo y puertos: desafíos y oportunidades en busca de un desarrollo sostenible en América Latina y el Caribe", serie Recursos Naturales e Infraestructura, № 176 (LC/L.4119), Santiago, Comisión Económica para América Latina y el Caribe (CEPAL).

Schwab, K. (ed.) (2016), The Global Competitiveness Report 2016-2017, Ginebra [en línea] http://www3. weforum.org/docs/GCR2016-2017/05FullReport/TheGlobalCompetitivenessReport2016-2017_FINAL.pdf.

Schurer, S. y J. Yong (2012), "Personality, well-being and the marginal utility of income: what can we learn from random coefficient models?", SEF Working Paper, № 01/2012, Wellington, Victoria University of Wellington [en línea] http://researcharchive.vuw.ac.nz/handle/10063/2040.

Serebrisky, T. y otros (2016), "Exploring the drivers of port efficiency in Latin America and the Caribbean", Transport Policy, vol. 45, Amsterdam, Elsevier.

The Maritime Executive (2014), "Drewry: GDP vs TEUs", Fort Lauderdale, 8 de septiembre [en línea] https:// www.maritime-executive.com/article/GDP-vs-TEU-Drewry-2014-09-08.

UNCTAD (Conferencia de las Naciones Unidas sobre Comercio y Desarrollo) (s/fa), "Review of Maritime Transport (Series)" [en línea] y https://unctad.org/en/pages/publications/Review-of-Maritime-Transport(Series).aspx.

(s/fb), "UNCTADSTAT" [en línea] http://unctadstat.unctad.org.

Wooldridge, J. M. (2010), Econometric Analysis of Cross Section and Panel Data, Cambridge, MIT Press. (2003), "Cluster-sample methods in applied econometrics", The American Economic Review, vol. 93, № 2, Nashville, Asociación Estadounidense de Economía. 